For: Journal of Economic Entomology

(Household and Structural Insects section)
Corresponding author:

Chow-Yang Lee Urban Entomology Laboratory Vector Control Research Unit School of Biological Sciences Universiti Sains Malaysia 11800 Penang, Malaysia Email: chowyang@usm.my

\title{
Differences in climbing ability of Cimex lectularius and Cimex hemipterus (Hemiptera: Cimicidae)
}

\author{
DAE-YUN KIM ${ }^{1}$, JOHAN BILLEN ${ }^{2}$, STEPHEN L. DOGGETT ${ }^{3}$ AND CHOW-YANG \\ $\mathrm{LEE}^{1,4}$ \\ ${ }^{1}$ Urban Entomology Laboratory, Vector Control Research Unit, School of Biological Sciences, \\ Universiti Sains Malaysia, 11800 Penang, Malaysia. \\ ${ }^{2}$ KU Leuven, Zoological Institute, Naamsestraat 59, box 2466, B-3000 Leuven, Belgium. \\ ${ }^{3}$ Department of Medical Entomology, Westmead Hospital, Westmead NSW 2145, Australia. \\ ${ }^{4}$ Corresponding author. Email: chowyang@usm.my
}

Running title: Kim et al. Climbing ability of Cimex lectularius and C. hemipterus 
ABSTRACT The climbing abilities of two bed bug species, Cimex lectularius L. and Cimex hemipterus (F.) were determined by evaluating their escape rates from smooth surface pitfall traps using four commercial bed bug monitors (Verifi ${ }^{\mathrm{TM}}$ Bed Bug Detector, ClimbUp ${ }^{\circledR}$ Insect Interceptor, BlackOut ${ }^{\mathrm{TM}}$ Bed Bug Detector, and SenSci Volcano ${ }^{\mathrm{TM}}$ Bed Bug Detector). All detectors were used in the absence of lures or attractants. Unlike $C$. lectularius, adult $C$. hemipterus were able to escape from all traps. On the other hand, no or low number nymphs of both species escaped, depending on the evaluated traps. Examination on the vertical friction force of adults of both species revealed a higher vertical friction force in $C$. hemipterus than in C. lectularius. Scanning electron micrograph observation on the tibial pad of adult bed bugs of $C$. hemipterus showed the presence of a greater number of tenent hairs on the tibial pad than on that of adult C. lectularius. No tibial pad was found in the fourth and fifth instars of both species. Near the base of the hollow tenent hairs is a glandular epithelium that is better developed in adult $C$. hemipterus than in adult $C$. lectularius. This study highlights significant morphological differences between C. lectularius and C. hemipterus, which has profound implications in the monitoring and potential management of bed bug infestations.

Key Words bed bug, tenent hair, vertical friction force, climbing, pitfall trap, monitoring, management.

\section{Introduction}

The current global resurgence of bed bugs involves two species, namely the common bed bug, Cimex lectularius L. and the tropical bed bug, Cimex hemipterus (F.). Cimex lectularius is mainly found in the temperate and subtropical regions, while the latter species is distributed in the subtropical and tropical regions (Omori 1939, 1941, Usinger 1966, Doggett et al. 2003, How and Lee 2010, Zulaikha et al. 2016). In some regions such as in Africa (Newberry 1988, 
1989), Australia (Doggett et al. 2003), Florida (Hixson 1943, Campbell et al. 2016), and Taiwan (Lee 2013), both species can co-exist sympatrically.

The detection and monitoring of bed bugs is one of the most important aspects of integrated pest management (IPM) against this insect (Wang \& Copper 2011). Detection and monitoring of bed bugs can be undertaken via visual inspections, through various traps and monitoring devices, via canine detection (Pinto et al. 2007, Wang \& Copper 2011, Boase \& Naylor 2014), or any combination of these. Amongst the monitors available in the market are pitfall and sticky traps, some with the addition of lures such as heat, carbon dioxide, and/or insect/host odors (active monitors), while others contain no lure (passive monitors) and provide a harborage for bed bugs to enter. While many such products exist on the market, few have been tested for efficacy via independent scientific tests.

Pitfall style traps of different designs have been found effective in detecting and monitoring $C$. lectularius (Singh et al. 2013). These traps rely on a smooth inner wall surface to prevent trapped bed bugs from escaping. Such traps with smooth surfaces can also be used as a barrier to prevent bed bugs from reaching the sleeping hosts (Doggett 2013). However, if the trapped bugs were able to climb up the smooth surfaces and escape, this would seriously compromise monitoring efforts or their use as barriers. It is known that a buildup of dust and debris can compromise the efficacy of pitfall traps against $C$. lectularius and hence maintenance recommendations are usually included such as regular cleaning or the addition of talc (Doggett 2013).

Most of the trap products were originally developed and tested in the U.S. where $C$. lectularius is the major bed bug species. Many of these products have subsequently been 
marketed in other parts of the world on the assumption that they also would work against the species that were found there, such as $C$. hemipterus. In a preliminary laboratory investigation though, it was observed that adult $C$. hemipterus could escape from a new pitfall trap, which otherwise effectively contained C. lectularius. This raised an important question: are all pitfall traps that are effective in containing $C$. lectularius also effective against $C$. hemipterus? If differences are found, this would have profound implications for the monitoring of the latter species and for the potential use of barriers against $C$. hemipterus. Furthermore, it would raise the question why such differences exist. The results of the initial investigation prompted the following study. The escape rate was compared between $C$. lectularius and $C$. hemipterus in four commercially available monitors that were previously or presently marketed for bed bug detection and monitoring in the US. Following this, the vertical friction force was determined for both species on two types of surfaces (smooth and rough). Lastly, the leg morphology was investigated to determine the possible reasons behind the differences in climbing ability of both species.

\section{Materials and Methods}

Insects. Two strains for each species were used; C. lectularius (Monheim [MH] and Sydney [SYD] strains), and C. hemipterus (Kuala Lumpur [KL] and Green lane [GL] strains) (Table 1). All strains were reared in the laboratory in glass jars (7 cm diameter $x 9 \mathrm{~cm}$ height) and provided with folded brown paper as harborage under environmental conditions of $26 \pm 2^{\circ} \mathrm{C}$, $70 \pm 5 \% \mathrm{RH}$, and a 12-h photo period. All the insects used in this study were blood-fed on a human volunteer once a week. An alcohol-preserved sample of adult $C$. hemipterus collected from Papua New Guinea in 1932 [PNG strain] was used in part of the scanning electron microscope (SEM) observation to examine if any morphological change could have been a recent evolutionary adaptation due to human management interventions. 
Trap escape test. The insects were evaluated in their ability to escape from four bed bug pitfall traps namely, Verifi ${ }^{\mathrm{TM}}$ bed bug detector (FMC Professional Solutions, Philadelphia, PA), ClimbUp® insect interceptor (Susan McKnight Inc, Memphis, TN) (without the talc), BlackOut $^{\mathrm{TM}}$ bed bug detector (Protect-A-Bed, Wheeling, IL), and SenSci Volcano ${ }^{\mathrm{TM}}$ bed bug detector (Bedbug Central, Lawrenceville, NJ). All traps were cleaned with 70\% ethanol and dried in a $60^{\circ} \mathrm{C}$ incubator overnight before the test. While ClimbUp ${ }^{\circledR}$ traps are normally used with the talc (as per manufacturer recommendation), they were evaluated in the absence of talc in this study. Lures are available for use in conjunction with the Verifi and Volcano traps to enhance their collection efficacy but they were not used in this study. One strain of each species was tested: C. lectularius (SYD strain) and C. hemipterus (KL strain). The evaluation was carried out by introducing 5 adult males, 5 adult females and 5 fourth to fifth instars into the trap. The tested insects were placed in the pitfall for both Verifi and Volcano traps, and in the outer pitfall for the ClimbUp and Blackout traps. The traps were placed inside test arenas (50 length x $30 \mathrm{~cm}$ width x $10 \mathrm{~cm}$ height) and kept in an environmental chamber (Binder Model KBF 240, Binder GmbH, Tuttlingen, Germany) under conditions of $26 \pm 1^{\circ} \mathrm{C}, 70 \pm 1 \%$ insects that remained in the trap, and the number that escaped were counted. Observations were made up to 72 hours for all traps except for the Volcano trap which was observed daily for up to 10 days post-treatment. All experiments were replicated three times.

Vertical friction force measurement. To confirm that the differences in climbing ability of the insects within the pitfall traps was due to better holding ability on the smooth surface of 
one species over the other, the vertical friction forces of both $C$. lectularius and $C$. hemipterus were measured with a Sartorius ED224S (Sartorius, Göttingen, Germany) electronic balance using modified methods described by Betz (2002) and Hottel et al. (2015). A data recording program, Sartorius Weight Anchor Software (Sartorius, Göttingen, Germany) installed on a computer, was connected to the electronic balance using a Sartorius RS232 cable (Sartorius, Göttingen, Germany). A bed bug was tethered to a polyethylene bristle by gluing (UHU Power Glue ${ }^{\circledR}$, UHU GmbH \& Co. KG, Bühl, Germany) its dorsal side to one tip of the bristle. The other tip of the bristle was inserted into a 30-g ball of modelling clay (Tack-it, A.W. Faber-Castell (India) Ltd, Mumbai, India). The clay ball, which was attached to the bristle, was placed on the balance with the tethered bed bug over the other end, and tared to zero.

Two surfaces were tested, namely a smooth surface (glass microscope cover slide), and a rough surface (filter paper). The test surface was moved to the tethered bed bug to permit the insect's legs to come into contact. Once the insect had contacted and gripped onto the test surface, the software started recording the changes in the mass of the clay ball for $5 \mathrm{~min}$. The data was then converted from mass $(\mathrm{g})$ to force $(\mu \mathrm{N})$ using the formula $F=m a$, where $m=$ mass (g), and $a=$ acceleration $=-9.81 \mathrm{~m}^{2} / \mathrm{s}$. The mean vertical friction force $(\mathrm{MVFF})$ was calculated using the top five maximum readings. One strain of each species was tested: $C$. lectularius (SYD strain), C. hemipterus (KL strain). Five adult males and five adult females were used for each species. All these insects were blood-fed 5 days prior to the test. The weight of the insects (g) was individually measured using the balance, and converted to $\mu \mathrm{N}$ using the formula as stated above. The MVFF: insect weight ratio was calculated and used to compare between both species, instead of using MVFF to equalize the effect of weight differences between the species. 
SEM observation of the tibial pad (fossula spongiosa). Five adult males and five adult

females of the two species (C. lectularius [MH and SYD strains], and C. hemipterus [KL, GL, and PNG strains]) were used for SEM observation of the tibial pad. Two each of $4^{\text {th }}$ instars and $5^{\text {th }}$ instar of $C$. lectularius (MH strain) were examined for the presence of the tibial pad. Bed bugs were preserved in 70\% ethanol, dehydrated and cleaned using acetone and a Sonicator SC-52H (Sonicor Instrument Corp, New York, US). The fore-, mid- and hind-legs of the specimens were excised and mounted on an aluminium stub. The specimens were coated with gold and observed under a scanning electron microscope (SEM) at $15 \mathrm{kV}$ (Leo Supra 50VP field emission SEM, Carl Zeiss SMT, Oberkochen, Germany). The number of tenent hairs (setae) on the tibial pad of each leg was counted from the SEM images taken from different angles.

Histological examination of the tibial pad in C. lectularius and C. hemipterus. The tibiae with attached tarsomeres of the six legs of three male and three female adults of both $C$. lectularius (MH strain) and C. hemipterus (QSL strain) were cut off with microsurgery scissors, and fixed in cold 2\% glutaraldehyde, buffered with 50 mM Na-cacodylate and 150 mM saccharose. Tissues were postfixed in $2 \%$ osmium tetroxide in the same buffer, dehydrated through a graded acetone series and embedded in Araldite resin. Serial semithin sections with a thickness of $1 \mu$ m were made with a Leica EM UC6 ultramicrotome (Leica Biosystems, Nussloch, Germany), stained with methylene blue and thionin, and examined with an Olympus BX-51 light microscope (Olympus Corporation, Tokyo, Japan). 
177 Statistical Analysis. Data in percentage of escaped bed bugs from the traps were converted to arc-sine values before the analyses. $T$-test was used to compare between the trap escape rates of $C$. lectularius and C. hemipterus. Data on vertical friction force, and the number of tenent hairs for adult males and females of both species were subjected to one-way Analysis of Variance (ANOVA) and means were separated using Tukey’s HSD at 95\% confidence level. All analyses were performed using the statistical program SPSS version 20 (IBM Corp., Armonk, NY).

\section{Results}

Trap escape test. Results showed that all stages tested of $C$. hemipterus were able to escape from each of the traps, with mean combined escape rates (\%) ranging from $24.4 \pm 4.4$ to 75.6 \pm 4.4 (Figure 1) per trap, at 72 hours post-treatment. In contrast, most traps were effective in containing C. lectularius with only 0 to $2.2 \%$ escape rates, except for the ClimbUp insect interceptor which registered a $26.7 \pm 10.2 \%$ mean escape rate. The escape rates from all traps were significantly different $(\mathrm{P}<0.05)$ between $C$. lectularius and $C$. hemipterus. For the Volcano trap, an observation of up to 10 days revealed an escape rate of $55.0 \pm 27.5 \%$ for $C$. hemipterus, while no C. lectularius escaped during the corresponding period.

When the monitors were tested against adults of $C$. hemipterus, the mean cumulative percentage of escaped insects in Verifi and ClimbUp traps were significantly higher $(\mathrm{P}<0.05)$ than the BlackOut and the Volcano traps at 4 hours post-treatment (Figure 2). Regarding $C$. lectularius, significantly $(\mathrm{P}<0.05)$ more adults escaped from the ClimbUp trap than from the other three evaluated traps. No nymphs of either species were able to escape from the Blackout and Volcano traps (Figure 2). For C. lectularius, no nymphs escaped from Verifi trap, but $13.3 \pm 6.7 \%$ nymphs escaped from ClimbUp trap at 72 hour post-treatment. When 
tested against adult $C$. lectularius, $3.3 \pm 3.3 \%$ and $33.3 \pm 12.0 \%$ adults escaped from Verifi and ClimbUp traps, respectively. Despite some nymph escapees, there were no significant differences $(\mathrm{P}>0.05)$ in the mean cumulative percentage of escaped nymphal bed bugs of all four evaluated traps. Both Blackout and Volcano traps were effective in containing the $C$. lectularius adults with no escapees throughout the 72 hours evaluation period (as well as at 10 days post-treatment for Volcano trap).

Nymphs of C. hemipterus managed to escape from Verifi and ClimbUp traps at the rates of $26.7 \pm 13.3 \%$ and $20.0 \pm 11.5 \%$, respectively at 72 hours. There were no nymph escapees for the Blackout and Volcano traps. Verifi was the least efficient trap against the adults of $C$. hemipterus with 100\% escapees within 4 hours after the bed bugs were introduced into the trap. ClimbUp trap also showed a high escape rate (>90\%) for C. hemipterus adults at 72 hour post-treatment. In contrast, $60.0 \pm 15.3$ and $36.7 \pm 6.7 \%$ of adult $C$. hemipterus escaped from Blackout and Volcano traps, respectively, during the same 72-hour evaluation period.

Vertical friction force. Visual observation revealed that when bed bugs were climbing on the rough surface, the tarsal claws were used, while tibial pads only were used while climbing on smooth surfaces (Figure 3). In this study, C. hemipterus demonstrated a significantly higher vertical friction force $(\mathrm{P}<0.05)$ of approximately $3-4 \mathrm{x}$ that of $C$. lectularius when tested on smooth glass microscope cover slides (Table 2). However, there appeared to be no differences in vertical friction force in both species when the insects were tested on filter paper as a rough substrate. No differences in vertical friction force were found between adult males and females of each species. 
SEM observation on tibial pad. Tibial pads were present in the adults of both species, but they were not present in the fourth instar (Figure 4) and fifth instar. The pads appear as an oval disk of approximately $120 \times 40 \mu \mathrm{m}$ at the ventrodistal portion of the tibia of the 6 legs (Figure 5). The ventral side of the pad is covered with parallel tenent hairs that have a golf club-like appearance. The hairs have a length between 30 and $40 \mu \mathrm{m}$ and a diameter around 2 $\mu \mathrm{m}$, the club-like distal portion having a length of approximately $10 \mu \mathrm{m}$ (Figure 5). The number of tenent hairs on the tibial pad of $C$. hemipterus was significantly higher $(\mathrm{P}<0.05)$ than in C. lectularius (Table 3, Figure 5). The number of tenent hairs appeared to be similar among the different strains of each species (Table 3), however, the fore- and mid-legs showed more tenent hairs than the hind-legs. The three strains of $C$. hemipterus have almost twice the number of tenent hairs $(\mathrm{P}<0.05)$ on the hind-legs, compared to $C$. lectularius. The adult male of C. lectularius had significantly more $(\mathrm{P}<0.05)$ tenent hairs than the adult female of the same species on their fore- and mid-legs, but this observation was not recorded for $C$. hemipterus. The rank in total tenent hairs on the fore-, mid- and hind-legs for one side of the body is as follows: $C$. hemipterus males $=C$. hemipterus females $>C$. lectularius males $>C$. lectularius females.

\section{Histological examination of tibial pad in C. lectularius and C. hemipterus. Serial}

longitudinal sections through the distal tibial part revealed details of the internal anatomy of the tibial pad region (Figure 6A - 6D). The tenent hairs are hollow with an internal diameter around $1 \mu \mathrm{m}$, and penetrate the cuticular plate that forms the base of the tibial pad. In all legs of both males and females of $C$. lectularius and C. hemipterus, the tegumental epithelium of the tibia is differentiated into a glandular epithelium. This epithelium in all legs is more pronounced in $C$. hemipterus, where it reaches a thickness of $30 \mu \mathrm{m}$, while in C. lectularius it is approximately half as thick (Figure 6A - 6D). At its apical side, the epithelium shows a 
subcuticular space in which its secretory products can be stored, and which is continuous with the central space of the hollow tenent hairs. The epithelium is not associated with any muscular tissue. The tibia contains two tendons, however, of which the ventral one passes in the near vicinity of the glandular epithelium. Both tendons are connected with muscles in the proximal part of the tibia and distally to the basitarsus, of which they direct the movements.

\section{Discussion}

Unlike C. lectularius, adult C. hemipterus were found to escape from all pitfall traps evaluated. Examination of the vertical friction force of both species revealed higher vertical friction force in C. hemipterus, compared to C. lectularius. As bed bugs climb on rough surface using their tarsal claws, and on smooth surfaces using the tibial pads, it was suspected that the differences in climbing ability between the two species must be related to variations in the tibial pad. Although Wigglesworth (1938) previously reported that the tibial pad did not assist in the climbing of smooth surfaces by the bed bug, he was experimenting using only $C$. lectularius. Should he have worked on C. hemipterus, his conclusion would have been very different. In the reduviid kissing bug (Triatoma spp.), it has been proposed that the tibial pads enable the adult bugs to climb smooth surfaces (Gillett \& Wigglesworth 1932, Weirauch 2007). Usinger (1966) described the presence of the tibial pad in C. lectularius, but did not indicate its presence in $C$. hemipterus.

To date, the tibial pad had been reported as “fossula spongiosa” by Haridass \& Ananthakrishnan (1980), “apex of tibia” by Walpole (1987), and “tibial brush” by several authors (Ferris \& Usinger 1957, Baker et al. 2016). The tenent hairs (Haridass \& Ananthakrishnan 1980) were described as a "turf of hair” (Usinger 1966), "tibial brush setae” (Baker et al. 2016), or “setae of tibial apex” (Walpole 1987). 
SEM observation on the tibial pad revealed a greater number of tenent hairs in $C$. hemipterus

277

than in C. lectularius. By incapacitating the function of tenent hairs on the tibial pad using glue, it was found that this affected the mating process of $C$. hemipterus, which led to a reduction in reproductive success (Kim \& Lee, unpublished data). It is speculated that the tibial pad may play an important role in the reproduction of the bed bug, beyond that of providing grip in the climbing of smooth surfaces. This argument is further supported by the absence of tibial pads in the nymphal stages, which explains their poor climbing ability as documented in this study. Using SEM, Walpole (1987) too reported that the tibial pad could only be found in the adults of C. lectularius and C. hemipterus, but failed to determine its function. In other insect orders such as Blattodea (Clemente \& Federle 2008), Diptera (Bauchhenss 1979, Gorb 1998, Gorb \& Beutel 2001), Hymenoptera (Dirks \& Federle 2011), Coleoptera (Ishii 1987, Geiselhardt et al. 2011, Hosoda \& Gorb 2011), as well as other families in the order Hemiptera (Gillett \& Wigglesworth 1932, Edwards \& Tarkanian 1970, Haridass \& Ananthakrishnan 1980), the use of the tenent hairs for surface adhesion has been documented. Baker et al. (2016) described the tenent hairs on the tibia pad as "tibia brush setae” and also suggested that these setae may be functional during the mating process and for climbing of various surfaces.

Although the precise mechanism of how the tibial pad allows climbing on smooth vertical surfaces remains still unknown, it seems obvious that the hollow tenent hairs play a role in the ability to do so. Their central space is continuous with the subcuticular space of the glandular epithelium that is associated with the tibial pad (Baker et al. 2016), suggesting that a glandular secretion can be released through the hairs. With an internal diameter around $1 \mu \mathrm{m}$, mere capillary action may be sufficient for this. In addition to capillarity, secretion may also 
be pumped through the hollow hairs, although there is no direct muscular tissue associated with the glandular epithelium. However, there is the ventral tibial tendon, that is situated in close proximity to the epithelium, and which may cause a pressure onto the epithelium and hence also on the fluid in the subcuticular space. Such pulling action of the tendon may occur during climbing when the tarsomeres need to be moved in order to bring the tibial pad in the right configuration with respect to the substrate. Increased pressure from the tendon onto the glandular epithelium at the same time may then result in fluid being pumped into the tenent hairs. A somewhat comparable situation is known in ants, in which adhesion to smooth surfaces is possible through the arolium that acts as an adhesive pad, which is situated at the tip of the pretarsus of each leg. The arolium is associated with a sac-like epithelial gland, that works as a hydraulic system by pumping liquid into the arolium upon contraction of the leg tendon (Federle et al. 2001). In a recent study comparing two ant species with very opposite climbing ability, it was found that both species possessed an arolium gland, although it was only poorly developed in the non-climbing species (Billen et al. 2016). These reports are in line with the observations herein that both Cimex species possess a tibial pad, but that the higher number of tenent hairs and the better developed glandular epithelium in C. hemipterus allow this species to climb smooth vertical surfaces.

In the experiments described herein, the ClimbUp trap was evaluated without the addition of talc, and C. hemipterus was able to easily escape from the monitor. When talc was applied (as per manufacturer recommendations), the monitor was able to contain insects of both $C$. lectularius and C. hemipterus with minimal escapees (<5\%) over a 72 hour period (Kim \& Lee, unpublished data). Wang \& Cooper (2011) reported that regular talc powder reapplications on pitfall-type monitors are required to prevent bed bugs from escaping. Hottel et al. (2015) found that talc powder reduced the vertical pulling force of C. lectularius on 
glass surfaces and the ClimbUp inner wall. In addition, from our experience, the number of trapped escapees could be minimized by placing a small piece of folded corrugated cardboard inside the pitfall trap. However, this would require further efforts as the harbourage would need to be examined during monitoring programs. It is anecdotally known that the performance of pitfall traps declines in use over time due to the build-up of dust and debris, and can even overcome the addition of talc (Singh et al. 2013, Wang et al. 2013, Cooper et al. 2016). A trial examining variably aged traps could provide insights into the required maintenance intervals to prevent escape of the respective species.

The present study demonstrated the need for the design of a more efficient bed bug trap (and the need to reconsider trap maintenance intervals) that could be used against both $C$. lectularius and $C$. hemipterus. New low friction surfaces for pitfall traps could also be explored, as long as the cost per unit item for the trap would not financially compromise any monitoring program. It remains unknown at this stage why these sympatric species of bed bugs could have these differences in leg morphology, and the possible biological trade-offs it may have on C. hemipterus.

\section{We thank Jamilah Afandi and Johari Othman (Electron Microscopy Unit, Universiti Sains} Malaysia) for assistance with scanning microscopy, An Vandoren (KU Leuven) for making the histological sections, and Annie Eow for helping with the field collection of $C$. hemipterus. This study was supported by Bayer Environmental Science, Singapore.

\section{Acknowledgments}


Baker, G.T., J. Goddard, and A. Lawrence. 2016. Structure of the tibial brush setae of the bedbug, Cimex lectularius L. (Hemiptera: Cimicidae). Poster presentation. Microscopy and Microanalysis 2016. July 24 - 28, 2016. Columbus, Ohio, USA. http://www.microscopy.org/MandM/2016/program/abstracts/PDP-24.pdf (retrieved on August 8, 2016).

Bauchhenss, E. 1979. Die Pulvillen von Calliphora erythrocephala (Diptera, Brachycera) als Adhäsionsorgane. Zoomorphologie 93: 99-123.

Betz, O. 2002. Performance and adaptive value of tarsal morphology in rove beetles of the genus Stenus (Coleoptera, Staphylinidae). J. Exp. Biol. 205: 1097-1113.

Billen, J., M.S. Al-Khalifa and R.R. Silva. 2016. Pretarsus structure in relation to climbing ability in the ants Brachyponera sennaarensis and Daceton armigerum. Saudi J. Biol. Sci. (in press) http://dx.doi.org/10.1016/j.sjbs.2016.06.007

Boase, C., and R. Naylor. 2014. Bed bug management. pp. 8-22. In: Urban insect pests: sustainable management strategies (P. Dhang, ed.), CABI Press, London, UK.

Campbell, B.E., P.G. Koehler, L.J. Buss \& R.W. Baldwin. 2016. Recent documentation of the tropical bed bug (Hemiptera: Cimicidae) in Florida since the common bed bug resurgence. Fla. Entomol. 99: 549-551.

Clemente, C.J., and W. Federle. 2008. Pushing versus pulling: Division of labour between tarsal attachment pads in cockroaches. Proc. R. Soc. B: Biol. Sci. 275:1329-1236.

Cooper, R.A., C. Wang, and N. Singh. 2016. Evaluation of a model community-wide bed bug management program in affordable housing. Pest Manag. Sci. 72 : 45 - 56.

Doggett, S.L. 2013. A Code of Practice for the Control of Bed Bug Infestations in Australia, 4th ed. Department of Medical Entomology, Westmead Hospital, Sydney, and The Australian Environmental Pest Managers Association. 
Doggett, S.L., M.J. Geary, W.J. Crowe, P. Wilson, and R.C. Russell. 2003. Has the tropical bed bug, Cimex hemipterus (Hemiptera: Cimicidae), invaded Australia? J. Environ. Health 3: 80-82.

Edwards, J.S., and M. Tarkanian. 1970. The adhesive pads of Heteroptera: a reexamination. Physiol. Entomol. 45: 1-5.

Dirks, J.-H., and W. Federle. 2011. Fluid based adhesion in insects - principles and challenges. Soft Mat. 7: 11047-11053.

Federle, W., E.L. Brainerd, T.A. McMahon and B. Hölldobler. 2001. Biomechanics of the movable pretarsal adhesive organ in ants and bees. Proc. Natl Acad. Sci. USA 98: 62156220.

Ferris, G.F., and R.L. Usinger. 1957. Notes on and descriptions of Cimicidae (Hemiptera). Microentomol. 22: 1-37.

Geiselhardt, S.F., S. Geiselhardt, and K. Peschke. 2011. Congruence of epicuticular hydrocarbons and tarsal secretions as a principle in beetles. Chemoecol. 21: 181-186.

Gillett, J.D.,and V.B. Wigglesworth. 1932. The climbing organs of an insect, Rhodnius prolixus Stal (Heteroptera-Reduviidae). Proc. R. Entomol. Soc. London B 111: 364-376.

Gorb, S., and R. Beutel. 2001. Evolution of locomotory attachment pads of hexapods. Naturwissenschaften 88: 530-534.

Gorb, S.N. 1998. The design of the fly adhesive pad: distal tenent setae are adapted to the delivery of an adhesive secretion. Proc. R. Soc. B: Biol. Sci. 265: 747-752.

Haridass, E.T., and T.N. Ananthakrishnan. 1980. Functional morphology of the fossula spongiosa in some reduviids (Insecta - Heteroptera - Reduviidae). Proc. Ind. Acad. Sci. 89: 457-466.

Hixson, H. 1943. The tropical bedbug established in Florida. Records of Cimex hemiptera, F., in houses in several localities in 1938-42. Fla. Entomol. 26: 47. 
Hosoda, N., and S.N. Gorb. 2011. Friction force reduction triggers feet grooming behaviour in beetles. Proc. R. Soc. B: Biol. Sci. 278: 1748-1752.

Hottel, B., R. Pereira, S. Gezan, R. Qing, W. Sigmund, and P. Koehler. 2015. Climbing ability of the common bed bug (Hemiptera: Cimicidae). J. Med. Entomol. 52: 289-295.

How, Y.F., and C.Y. Lee. 2010. Survey of bed bugs in infested premises in Malaysia and Singapore. J. Vect. Ecol. 35: 89-94.

Ishii, S. 1987. Adhesion of a leaf feeding ladybird Epilachna vigintioctomaculata (Coleoptera: Coccinellidae) on a vertically smooth surface. Appl. Entomol. Zool. 22: 222228.

Lee, C.Y. 2013. Bed bugs in Asia - Perspective from Southeast Asia, Global Bed Bug Summit, 5-6 December 2013, Denver, Colorado.

Newberry, K. 1988. Production of a hybrid between the bedbugs Cimex hemipterus and Cimex lectularius. Med. Vet. Entomol. 2: 297-300.

Newberry, K. 1989. The effects on domestic infestations of Cimex lectularius bedbugs of interspecific mating with Cimex hemipterus. Med. Vet. Entomol. 3: 407-414.

Omori, N. 1939. Experimental studies on the cohabitation and crossing of two species of bedbugs (Cimex lectularius L. and C. hemipterus F.) and on the effects of interchanging of males of one species for the other, every alternate days, upon the fecundity and longevity of females of each species. Acta Jap. Medic. Tropic. 1: 127-154.

Omori, N. 1941. Comparative studies on the ecology and physiology of common and tropical bed bugs, with special reference to the reactions to temperature and moisture. J. Med. Assoc. Formosa 60: 555-729.

Pinto, L.J., R.A. Cooper, and S.K. Kraft. 2007. Bed bug handbook: The complete guide to bed bugs and their control. Pinto \& Associates, Inc. Mechanicsville, MD, USA. 
Singh, N., C. Wang, and R.A. Cooper. 2013. Effect of trap design, chemical lure, carbon dioxide release rate, and source of carbon dioxide on efficacy of bed bug monitors. J. Econ. Entomol. 106: 1802-1811.

Usinger, R.L. 1966. Monograph of Cimicidae (Hemiptera - Heteroptera). Entomological Society of America, College Park, Maryland.

Walpole, D. 1987. External morphology of the legs of two species of bed bugs (Hemiptera: Cimicidae). J. Entomol. Soc. South. Afr. 50: 193-201.

Wang, C. and R.A. Cooper. 2011. Environmentally sound bed bug management solutions. pp. 44 - 63. In: P. Dhang (ed.), Urban Pest Management: An Environmental Perspective. CABI, Oxon, UK.

Wang, C., N. Singh, R.A. Cooper, C.F. Liu, and G. Buczkowski. 2013. Evaluation of an insecticide dust band treatment method for controlling bed bugs. J. Econ. Entomol. 106: $347-352$.

Weirauch, C. 2007. Hairy attachment structures in Reduviidae (Cimicomorpha, Heteroptera), with observations on the fossula spongiosa in some other Cimicomorpha. Zool. Anz. 246: 155-175.

Wigglesworth, V. 1938. Climbing organs in insects. Nature 141: 974-975.

Zulaikha, Z., A.M.A. Hafiz, A.R.A. Hafis, and A.A. Hassan. 2016. A survey on the infestation levels of tropical bed bugs in Peninsular Malaysia: Current updates and status on resurgence of Cimex hemipterus (Hemiptera: Cimicidae). Asian Pac. J. Trop. Dis. 6: $40-45$. 
Table 1: The bed bug strains used in this study.

\begin{tabular}{llll}
\hline Species & Strain & $\begin{array}{l}\text { Year } \\
\text { established }\end{array}$ & Remarks \\
\hline & Greenlane (GL) & 2015 & field collected \\
Kuala Lumpur (KL) & 2005 & lab colony \\
$\begin{array}{l}\text { C. } \\
\text { hemipterus }\end{array}$ & Queensland (QSL) & $\sim 2004$ & lab colony \\
& $\begin{array}{l}\text { Papua New Guinea } \\
\text { (PNG) }\end{array}$ & 1932 & $\begin{array}{l}\text { curated specimens } \\
\text { in 70\% ethanol }\end{array}$ \\
& Monheim (MH) & & \\
C. & Sydney (SYD) & $\sim 1970$ & lab colony \\
& & 2004 & lab colony \\
\hline
\end{tabular}

446 
447 Table 2. Mean vertical friction forces (MVFF) on smooth and rough surfaces, weights 448 and MVFF: weight ratios of the adult male and female of $C$. lectularius and $C$. 449 hemipterus.

450

\begin{tabular}{|c|c|c|c|c|c|c|}
\hline \multirow{2}{*}{ Species } & \multirow{2}{*}{ Sex } & \multicolumn{2}{|c|}{$\mathrm{MVFF} \pm \mathrm{SE}(\mu \mathrm{N})^{1}$} & \multirow{2}{*}{ weight $(\mu \mathrm{N})$} & \multicolumn{2}{|c|}{ MVFF: weight ratio } \\
\hline & & Cover glass & Filter paper & & Cover glass & Filter paper \\
\hline \multirow{3}{*}{ C. hemiptert } & $\mathrm{M}$ & $156.8 \pm 9.2 \mathrm{a}$ & $2476 \pm 140.5 a$ & $25.5 \pm 2.5 c$ & $6.3 \pm 0.6 a$ & $102 \pm 13.4 \mathrm{a}$ \\
\hline & & & & & & \\
\hline & $\mathrm{F}$ & $184.8 \pm 37.8 \mathrm{a}$ & $3203 \pm 579.5 a$ & $30.6 \pm 2.0 \mathrm{bc}$ & $6.5 \pm 1.7 \mathrm{a}$ & $110 \pm 25.0 \mathrm{a}$ \\
\hline \multirow{3}{*}{ C. lectulari } & M & $48.7 \pm 10.2 b$ & $2338 \pm 307.3 a$ & $37.9 \pm 2.4 \mathrm{ab}$ & $1.3 \pm 0.3 b$ & $62 \pm 6.7 \mathrm{a}$ \\
\hline & & & & & & \\
\hline & $\mathrm{F}$ & $42.2 \pm 11.8 \mathrm{~b}$ & $3181 \pm 516.0 \mathrm{a}$ & $43.6 \pm 4.1 \mathrm{a}$ & $1.0 \pm 0.2 b$ & $77 \pm 17.9 a$ \\
\hline
\end{tabular}

$451 \quad{ }^{1}$ Means followed by different letters within the same column are significantly different $(\mathrm{P}<0.05$; 452 Tukey's HSD). 
Table 3. Mean number of tenent hairs on tibial pad of the fore-, mid- and hind-legs of adult $C$. hemipterus and $C$. lectularius.

\begin{tabular}{|c|c|c|c|c|c|c|}
\hline Species & Sex & Strains & Fore leg $^{1}$ & Mid leg ${ }^{1}$ & Hind leg $^{1}$ & Total $^{1}$ \\
\hline \multirow{6}{*}{ C. hemipterus } & \multirow{3}{*}{$\mathrm{M}$} & KL & $117.9 \pm 5.7 \mathrm{a}$ & $107.1 \pm 6.0 \mathrm{a}$ & $86.2 \pm 2.5 \mathrm{a}$ & $335.7 \pm 13.6 a$ \\
\hline & & GL & $101.8 \pm 2.6 \mathrm{ab}$ & $97.6 \pm 3.4 \mathrm{a}$ & $78.8 \pm 2.5 a$ & $292.2 \pm 9.2 \mathrm{ab}$ \\
\hline & & PNG & $113.1 \pm 2.9 \mathrm{a}$ & $106.4 \pm 2.7 \mathrm{a}$ & $79.3 \pm 2.4 a$ & $318.2 \pm 8.1 \mathrm{a}$ \\
\hline & \multirow{3}{*}{$\mathrm{F}$} & KL & $100.0 \pm 8.1 \mathrm{ab}$ & $112.7 \pm 3.1 \mathrm{a}$ & $88.1 \pm 1.9 \mathrm{a}$ & $309.7 \pm 11.8 \mathrm{a}$ \\
\hline & & GL & $105.7 \pm 4.3 a$ & $110.3 \pm 3.4 a$ & $87.7 \pm 2.9 a$ & $328.6 \pm 9.9 a$ \\
\hline & & PNG & $115.9 \pm 3.6 \mathrm{a}$ & $115.7 \pm 4.2 \mathrm{a}$ & $89.8 \pm 1.7 \mathrm{a}$ & $340.6 \pm 10.0 \mathrm{a}$ \\
\hline \multirow{4}{*}{ C. lectularius } & \multirow{2}{*}{$\mathrm{M}$} & MH & $119.8 \pm 7.3 a$ & $99.3 \pm 5.4 a$ & $41.8 \pm 2.2 b$ & $247.6 \pm 10.4 b c$ \\
\hline & & SYD & $114.2 \pm 5.3 a$ & $102.7 \pm 3.9 a$ & $49.2 \pm 2.0 b$ & $239.4 \pm 12.2 c$ \\
\hline & \multirow[b]{2}{*}{$\mathrm{F}$} & $\mathrm{MH}$ & $66.8 \pm 0.7 c$ & $71.6 \pm 1.4 b$ & $43.2 \pm 2.4 b$ & $176.8 \pm 4.0 \mathrm{~d}$ \\
\hline & & SYD & $79.6 \pm 1.7 \mathrm{bc}$ & $78.0 \pm 3.9 b$ & $47.8 \pm 1.9 b$ & $200.4 \pm 7.0 \mathrm{~cd}$ \\
\hline
\end{tabular}

${ }^{1}$ Means followed by different letters within the same column are significantly different $(\mathrm{P}<0.05$, Tukey's HSD).

460 


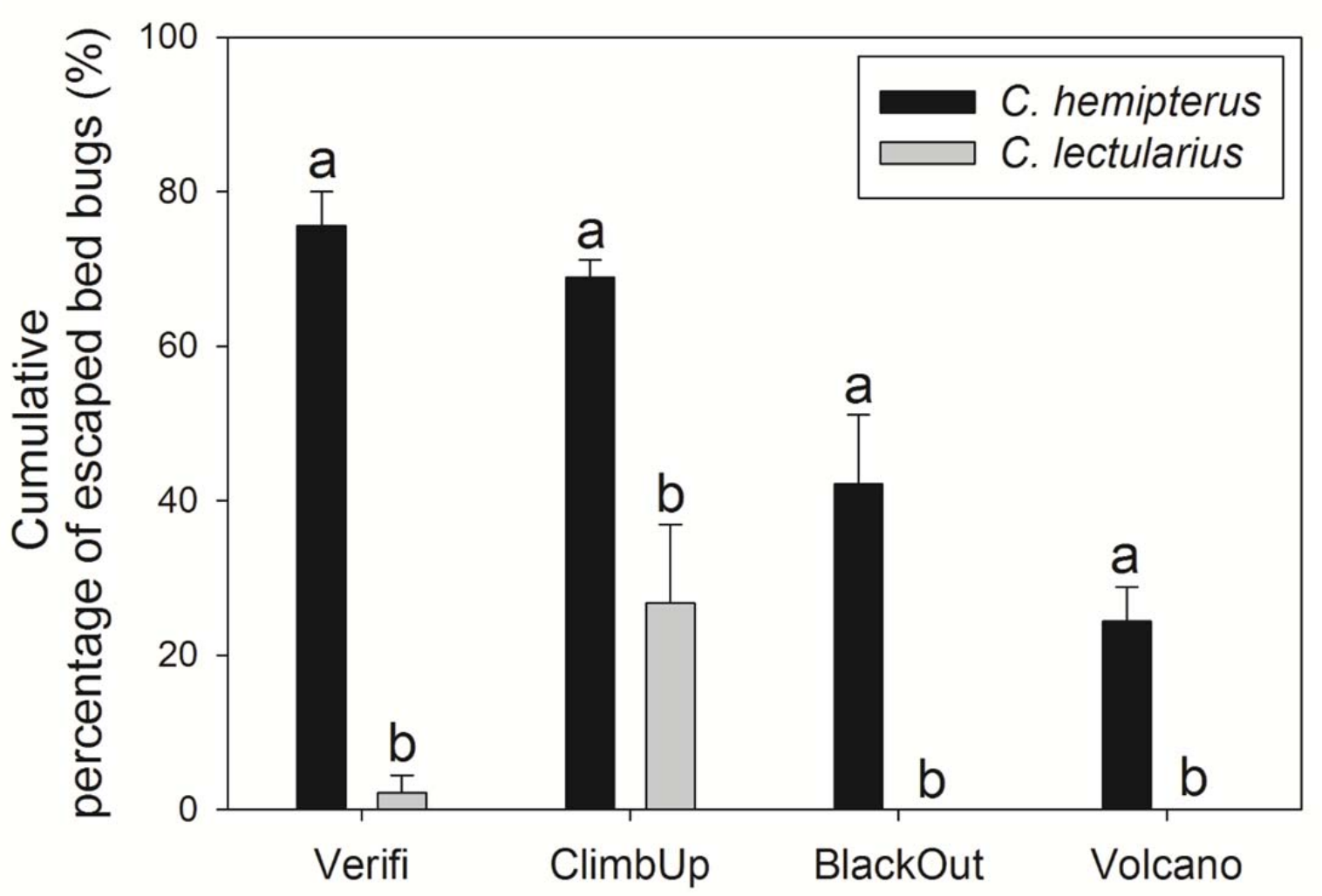

463

Fig. 1. Cumulative percentage of combined escaped adults and nymphs of bed bugs (Mean \pm SEM) from the four traps after 72-h post treatment. Letters above each bar are assigned for comparison between the same trap and different letters denote significant difference $(\mathrm{P}<0.05$, t-test).

468

469

470

471

472

473

474 

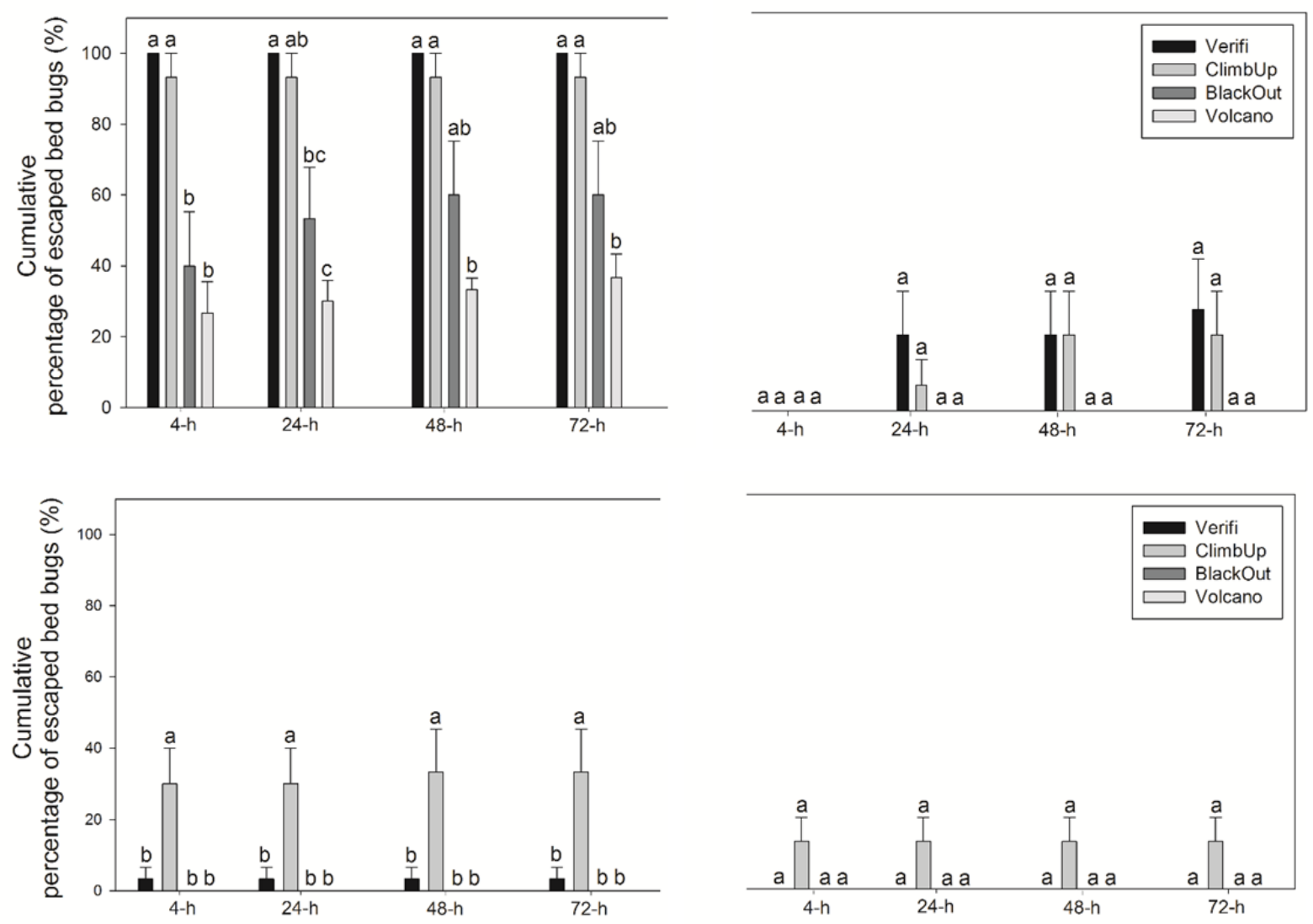

Fig. 2. Mean cumulative percentage of escaped bed bugs \pm SE at 4, 24, 48 and 72-h post treatment (Top left: $C$. hemipterus adults; Top right: $C$. hemipterus nymphs; Bottom left: $C$. lectularius adults; Bottom right: $C$. lectularius nymphs). Letters above each bar are assigned for comparison between the traps and different letters denote significant difference $(P=0.05$,
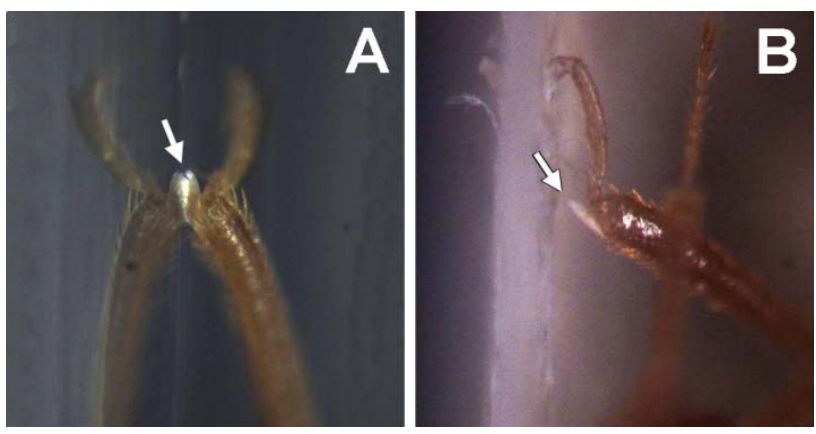

Fig. 3. Detail of foreleg of $C$. hemipterus male climbing on plastic petri dish (smooth surface) (A) and filter paper (rough surface) (B). Note that whitish tibial pad (arrow) is in contact with substrate only on smooth surface. Observation was made using live bed bugs under an SZ61 stereomicroscope (Olympus, Tokyo, Japan) connected to a CCD camera. 
487

488

489

490

491

492

493

494

495

496

497

498

499

500

501

502

503
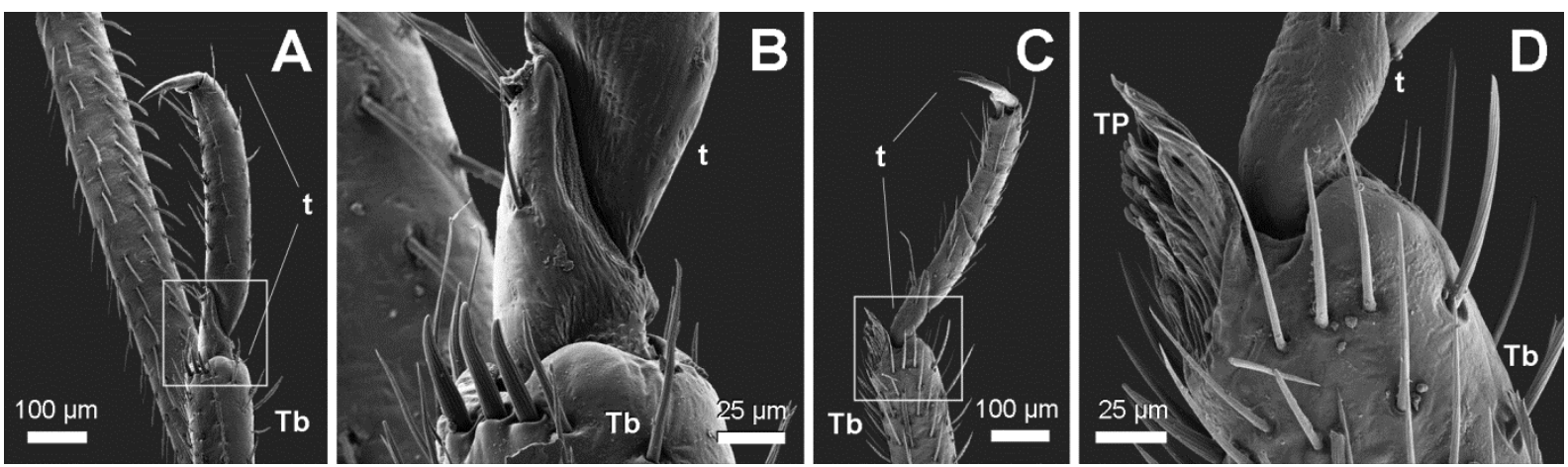

Fig. 4. SEM micrographs showing the absence of tibial pad in 4th instar $C$. lectularius (A, B), and presence of tibial pad in adult female $C$. lectularius $(\mathbf{C}, \mathbf{D})$. The framed area in A and C indicate the parts that are enlarged in $\mathrm{B}$ and $\mathrm{D}$, respectively. $\mathrm{t}$ : tarsomeres, Tb: tibia, TP: tibial pad.
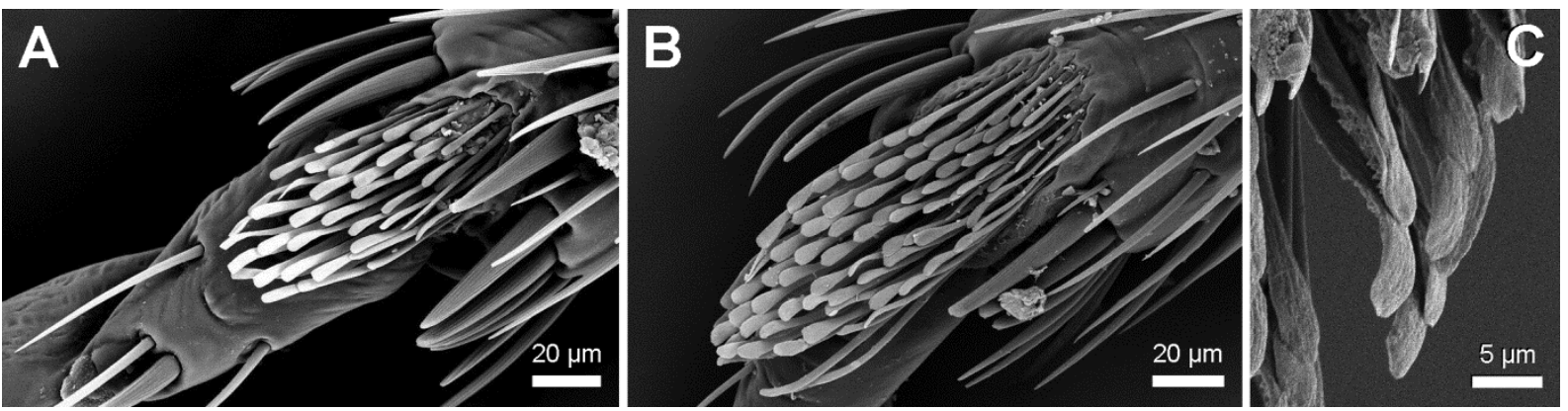

Fig. 5. SEM micrographs showing the tibial pad of the hind legs of an adult female in $C$. lectularius (A) and C. hemipterus (B). C. Detail of tenent hairs of male C. hemipterus mid leg.
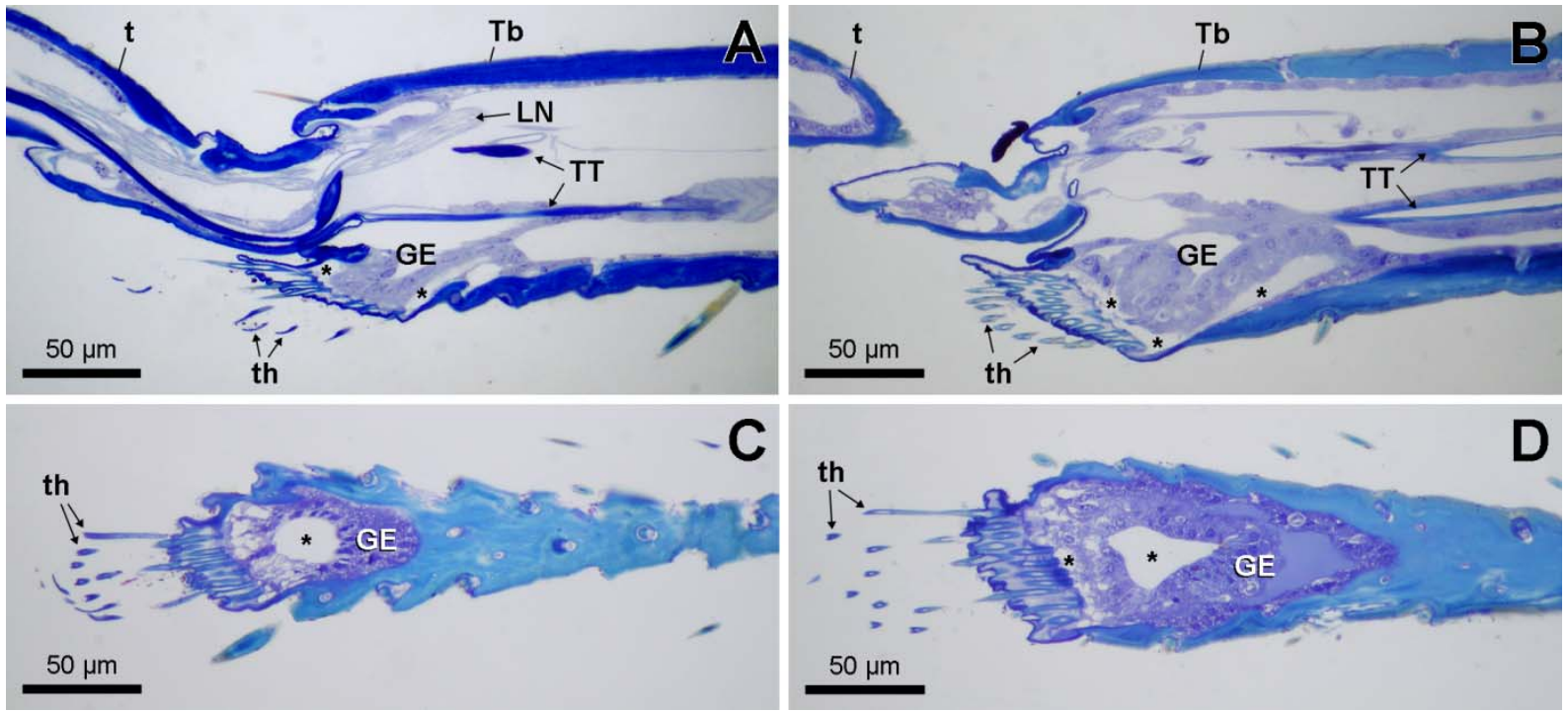

Fig. 6. Longitudinal sections through the tibial pad of $C$. lectularius (A, male mid leg) and $C$. hemipterus (B, male fore leg), showing the glandular epithelium (GE), the subcuticular space (asterisks) and the hollow tenent hairs (th). Note the tibia contains two tibial tendons (TT), of which the ventral is close to the glandular epithelium. The sectioning plane in $\mathbf{C}(C$. lectularius female hind leg) and $\mathbf{D}$ (C. hemipterus male mid leg) is perpendicular to that in $\mathrm{A}$ and $\mathrm{B}$, and is more or less parallel to the ventral surface of the tibia. LN: leg nerve, $t$ : tarsomeres, Tb: tibia. The histological organization in all legs is similar, the photographs shown in this figure were selected as they were the best available images. 